\title{
On Dualities Of Primordial Perturbation Spectra
}

\author{
Yun-Song $\mathrm{Piao}^{a, \text {, }}$ \\ ${ }^{a}$ Institute of High Energy Physics, Chinese Academy of Science, \\ P.O. Box 918-4, Beijing 100039, P. R. China and \\ ${ }^{b}$ Interdisciplinary Center of Theoretical Studies, Chinese Academy of Sciences, P.O. Box 2735, Beijing 100080, China
}

\begin{abstract}
In this paper, we discuss the dualities of the primordial perturbation spectra from various expanding/contracting phases for full space of parameter $w \equiv \frac{p}{\rho}$ of state equation.
\end{abstract}

PACS numbers: $98.80 . \mathrm{Cq}, 98.70 . \mathrm{Vc}$

The primordial perturbations on large scales may be an important test for various scenarios of the early universe, thus it is very significant to further probe their possible nature and origin. The basic idea of inflation is simple and elegant 1], for a recent review see [2]. During inflation, the fluctuations are stretched to the outside of horizon and form nearly scale-invariant primordial perturbations leading to the formation of cosmological structure [3]. Wands found 4] (see also [5], for an earlier reference see [6] ) that a collapsing universe with $a \sim(-t)^{\frac{2}{3}}$ ( further for Pre Big Bang-like scenario see [7, 8] ), in which $a(t)$ is the cosmological scale factor, can also generate such scale-invariant spectrum, which is dual to the case of inflation with $a \sim e^{h t}$ or $a \sim t^{n}, n \rightarrow+\infty$. This duality is a result that the spectrum of the curvature perturbation $\zeta$ on uniform comoving hypersurfaces is invariant under some transformation. Recently, it has been noted [9, 10] (see also 11, 12]) that instead of the curvature perturbation $\zeta$, when the Bardeen potential $\Phi$ is considered, the scale-invariant spectrum can be produced during a period of slow contraction with $a \sim(-t)^{n}, n \rightarrow 0_{+}$in cyclic scenario [13], for some criticisms see [5, 14, 15, 16]. This agreement between physically dissimilar models with different cosmological solutions is not coincidental. As has been showed further by Boyle et.al. [17], the relationship between inflation and cyclic scenario can be regarded as a special case of general duality of the perturbation spectrum of Bardeen potential $\Phi$.

For the parameter $w \equiv \frac{p}{\rho} \geq-1$ of state equation, Boyle et.al.'s duality relates a stable expanding solution to an stable/unstable contracting solution dependent on the value of $w$, while Wands's duality relates a stable expanding solution $(-1<w<-1 / 3)$ to an unstable contracting solution $(-1 / 3<w<0)$, but that of $w>$ 0 has not relevant dual branch, thus in some sense the dualities of perturbation spectrum may be uncomplete when restricted to $w>-1$. When relaxing this limit, the nearly scale-invariant perturbation spectrum can be also similarly obtained during slow expansion [18] and phantom inflation [19], but with different cosmological background evolutions $a \sim(-t)^{n}$, in which $n \rightarrow 0_{-}$and $n \rightarrow-\infty$ respectively. In this brief report, we discuss the

*Electronic address: yspiao@itp.ac.cn dualities of the primordial perturbation spectra for full space of constant $w$, which can be implemented in the frame of single normal/phantom scalar field [20].

In general the evolution of cosmological scale factor before the "bounce" 1 in Einstein frame can be written as

$$
a(t) \sim t^{n}
$$

in which $t \rightarrow+\infty$, or

$$
a(t) \sim(-t)^{n}
$$

in which $t \rightarrow 0_{-}, n$ is a positive or negative constant. For $n>0$, Eq. (11) corresponds to the expanding phase, in which the perturbation spectrum was studied firstly in Ref. [23], and Eq. (2) corresponds to the contracting phase. For $n<0$ the case is in reverse. The Fridmann equations are

$$
\begin{gathered}
h^{2} \equiv\left(\frac{\dot{a}}{a}\right)^{2}=\frac{\rho}{3} \\
\dot{h}=-\frac{\rho+p}{2}
\end{gathered}
$$

where $h$ is Hubble parameter and $8 \pi G=1$ is set. For the case that the speed of sound $c_{s}^{2}$ is constant, causally primordial perturbations can be generated in such a phase, in which the fluctuations exits the horizon and then reenters the horizon after the "bounce" to an expanding phase corresponding to our observational cosmology. This requires that $a h$ increases with time, thus $n>1$ for (11) and $n<1$ for (2) must be satisfied. From (11), (2) and (3), we have

$$
h=\frac{n}{t}
$$

for various phases. We plot the sketch of relations between $h$ and $t, n$ in Fig.1. These various expanding/contracting phases can be implemented by the evolution of single normal/phantom scalar field with exponential potential, in this case $c_{s}^{2}=1$ [24], which is summarized in Table I.

\footnotetext{
1 Here the "bounce" means the exit from pre-bounce expanding/contracting phases to late-time observational cosmology, which corresponds to the usual reheating 21, 22] for inflation scenario.
} 


\begin{tabular}{||l|l|l|l|l||}
\hline \hline Region & I & II & III & IV \\
\hline Evolution of scale factor & $a(t) \sim(-t)^{n}$ & $a(t) \sim t^{n}$ & $a(t) \sim(-t)^{n}$ & $a(t) \sim(-t)^{n}$ \\
\hline & $n<0$ & $n>1$ & $\frac{1}{3}<n<1$ & $0<n<\frac{1}{3}$ \\
\hline Hubble parameter & $h>0, h>0$ & $h>0, h<0$ & $h<0, h<0$ & $h<0, h<0$ \\
\hline & expansion & expansion & contraction & contraction \\
\hline Stability & stable & stable & unstable & stable \\
\hline $\begin{array}{l}\text { Parameter of state } \\
\text { equation }\end{array}$ & $w<-1$ & $-1<w<-\frac{1}{3}$ & $-\frac{1}{3}<w<1$ & $w>1$ \\
\hline$\epsilon$ & $\epsilon<0$ & $0<\epsilon<1$ & $1<\epsilon<3$ & $\epsilon>3$ \\
\hline Kinetic energy term & $\begin{array}{l}\text { reverse } \\
\text { (phantom) }\end{array}$ & standard & standard & standard \\
\hline Potential energy term & standard & standard & standard & $\begin{array}{l}\text { reverse (negative } \\
\text { potential) }\end{array}$ \\
\hline$\Phi$ invariant dual regions & I & III or IV & II & II or II or IV \\
\hline$\zeta$ invariant dual regions & III & III & \\
\hline \hline
\end{tabular}

TABLE I: The natures of various expanding and contracting phases generating the primordial perturbation spectra: These phases can be implemented by simple (normal/phantom) scalar field. Some further details can be seen in our paper [20].

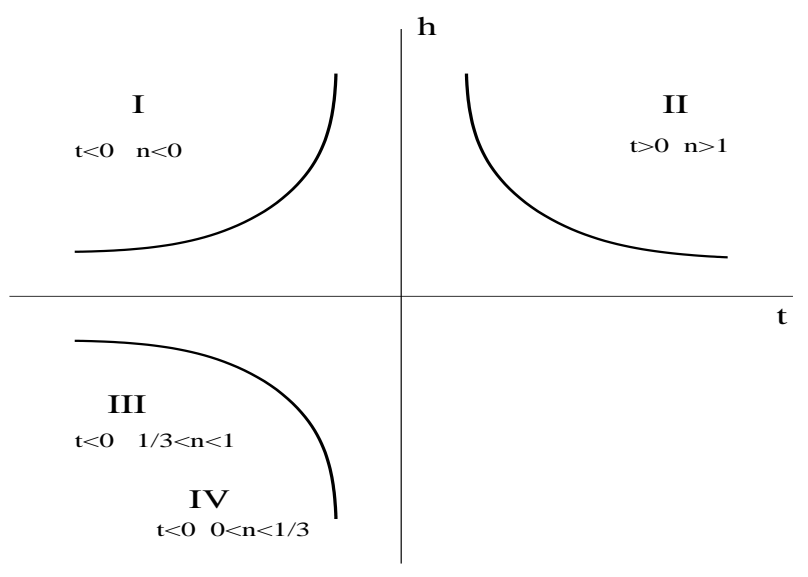

FIG. 1: The sketch of relations between Hubble parameter $h$ and $t, n$ : Various expanding/contracting phases are denoted by Region I-IV in Table I.

Combing (1), (2), (3) and (4), the power-law index of scale factor is given by

$$
n=\frac{2}{3(1+w)}
$$

Instead of $w$, it may be more convenient to parameterize the state equation with

$$
\epsilon \equiv \frac{3}{2}(1+w) \equiv \frac{1}{n}
$$

For $w \simeq-1, \epsilon \simeq 0$ is the usual slow-roll parameter of inflation models.

In the following we briefly review the primordial perturbation spectra from these expanding/contracting phases before the "bounce". Let us pay attention to the scalar metric fluctuations. In longitudinal gauge and in absence of anisotropic stresses, the scalar metric perturbation can be written as

$$
d s^{2}=a^{2}(\eta)\left(-(1+2 \Phi) d \eta^{2}+(1-2 \Phi) \delta_{i j} d x^{i} d x^{j}\right)
$$

where $\eta$ is conformal time $d \eta \equiv \frac{d t}{a}$, thus

$$
\begin{gathered}
-\eta \sim( \pm t)^{-n+1} \\
a(\eta) \sim(-\eta)^{\frac{n}{1-n}} \equiv(-\eta)^{\frac{1}{\epsilon-1}}
\end{gathered}
$$

and $\Phi$ is the Bardeen potential. The curvature perturbation on uniform comoving hypersurfaces is given by

$$
\zeta=\frac{1}{\epsilon}\left(\frac{a}{a^{\prime}} \Phi^{\prime}+\Phi\right)+\Phi
$$

After defining new variables 25, 26] (for a thorough introduction to gauge-invariant perturbations see [27, 28]),

$$
\begin{aligned}
& u=\frac{a}{\sqrt{2\left|\mathcal{H}^{2}-\mathcal{H}^{\prime}\right|}} \Phi=\frac{a}{\varphi^{\prime}} \Phi \\
& v=\frac{a \sqrt{2\left|\mathcal{H}^{2}-\mathcal{H}^{\prime}\right|}}{\mathcal{H}} \zeta=\frac{a \varphi^{\prime}}{\mathcal{H}} \zeta
\end{aligned}
$$

where $\mathcal{H} \equiv \frac{a^{\prime}}{a}$, the perturbation equations of $\Phi$ and $\zeta$ for simple (normal/phantom) scalar field can be written as

$$
\begin{aligned}
& u_{k}^{\prime \prime}+\left(k^{2}-\frac{\mu^{2}-\frac{1}{4}}{\eta^{2}}\right) u_{k}=0 \\
& v_{k}^{\prime \prime}+\left(k^{2}-\frac{\nu^{2}-\frac{1}{4}}{\eta^{2}}\right) v_{k}=0
\end{aligned}
$$

For all interesting modes $k$, we can solve Eqs. (14) and (15) analytically and obtain

$$
\begin{aligned}
& u_{k}=\sqrt{-k \eta}\left(B_{1}(k) J_{\mu}(-k \eta)+B_{2}(k) J_{-\mu}(-k \eta)\right) \\
& v_{k}=\sqrt{-k \eta}\left(C_{1}(k) J_{\nu}(-k \eta)+C_{2}(k) J_{-\nu}(-k \eta)\right)
\end{aligned}
$$


where

$$
\mu=\frac{1}{2}\left|\frac{\epsilon+1}{\epsilon-1}\right|, \quad \nu=\frac{1}{2}\left|\frac{\epsilon-3}{\epsilon-1}\right|,
$$

$J$ is the first kind of the Bessel function with order $\mu$ or $\nu$, the function $B_{i}(k)$ and $C_{i}(k)$ can be determined by specifying the initial conditions.

In the regime $k \eta \rightarrow \infty$, in which the mode $u_{k}$ and $v_{k}$ are very deep in the horizon, Eqs. (14) and (15) are reduced to the equations for a simple harmonic oscillator, in which $u_{k} \sim \frac{e^{-i k \eta}}{(2 k)^{3 / 2}}$ and $v_{k} \sim \frac{e^{-i k \eta}}{(2 k)^{1 / 2}}$ are stable. In the regime $k \eta \rightarrow 0$, in which the mode $u_{k}$ and $v_{k}$ are far out the horizon, the modes are unstable and grows. In longwave limit, $\Phi_{k}$ and $\zeta_{k}$ can be given and expanded to the leading term of $k$,

$$
\begin{aligned}
& k^{\frac{3}{2}} \Phi \sim k^{\frac{1}{2}-\mu} \\
& k^{\frac{3}{2}} \zeta \sim k^{\frac{3}{2}-\nu}
\end{aligned}
$$

Thus the spectrum indexes are

$$
\begin{aligned}
& n_{\Phi}-1=1-2 \mu=1-\left|\frac{\epsilon+1}{\epsilon-1}\right| \\
& n_{\zeta}-1=3-2 \nu=3-\left|\frac{\epsilon-3}{\epsilon-1}\right|
\end{aligned}
$$

From Eqs. (21) and (22), we can see that the the spectrum index of $\zeta$ is the same as of $\Phi$ only for $-1<\epsilon<1^{2}$, and when $\epsilon \simeq 0$, nearly scale-invariant can be obtained, which corresponds to the case in inflationary cosmology, but for other value of $\epsilon$, the spectrum index of $\zeta$ is different from that of $\Phi$, the $\Phi$ spectrum is nearly scaleinvariant for $\epsilon \rightarrow \pm \infty$, while the $\zeta$ spectrum is nearly scale-invariant for $\epsilon \simeq \frac{3}{2}$.

Further, notice from Eq. (18) that $\mu$ is invariant under $\frac{\epsilon+1}{\epsilon-1} \rightarrow-\frac{\epsilon+1}{\epsilon-1}$, which is equal to $\epsilon \rightarrow \frac{1}{\epsilon}$, in which the fixed points are $\epsilon= \pm 1$. Thus the spectrum index of $\Phi$ is invariant under this transformation, which can be also seen from Eqs. (19) and (21). Similarly, the spectrum index of $\zeta$ is invariant under the transformation of $\frac{\epsilon-3}{\epsilon-1} \rightarrow$ $-\frac{\epsilon-3}{\epsilon-1}$, i.e. $\epsilon \rightarrow \frac{2 \epsilon-3}{\epsilon-2}$, in which the fixed points are $\epsilon=$ 1,3 .

Therefore, for $\epsilon$ being in the range $0 \leq \epsilon<\infty$, as has been shown in Ref. [17], Wands's duality pairs the

\footnotetext{
${ }^{2}$ It is known that the comoving curvature perturbation $\zeta$ and the Bardeen potential $\Phi$ have different spectral dependence during the collapse and it is impossible for both them to obey nonsingular evolution equations through the bounce [30], see also Ref. [5, 31, 32, 33] and Ref. [20] for a relevant discussions. Which of the spectra of $\Phi$ and $\zeta$ can be inherited in late-time observational cosmology dependent on the matching conditions through the "bounce", i.e. how $\Phi$ and $\zeta$ pass through the "bounce", which is determined by the details of "bouncing" physics.
}

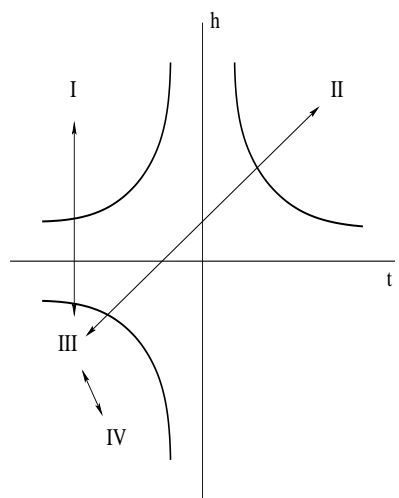

(a)

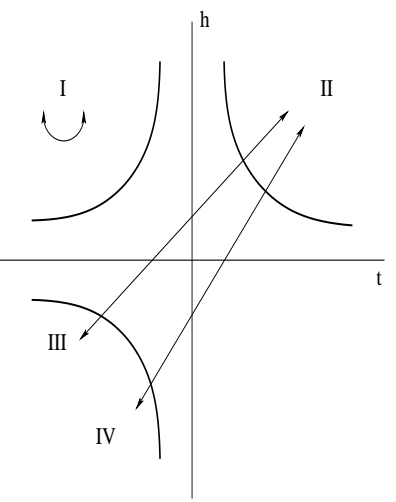

(b)
FIG. 2: The sketch of general dualities of $\zeta(\mathrm{a})$ or $\Phi(\mathrm{b})$ spectrum: Various expanding/contracting phases are denoted by

\begin{tabular}{|c|c|c|c|}
\hline \multicolumn{4}{|c|}{ The Dualities of Nearly Scale-Invariant Spectra } \\
\hline & \multicolumn{2}{|c|}{$\Phi$ spectrum } & $\zeta$ spectrum \\
\hline Phase & $\begin{array}{l}\text { (Phantom) Inflation } \\
\left(a \sim(-t)^{n}, n \rightarrow-\infty\right)\end{array}$ & $\begin{array}{c}\text { Inflation } \\
\left(a \sim t^{n}, n \rightarrow+\infty\right)\end{array}$ & Inflation \\
\hline $\begin{array}{l}\text { Dual } \\
\text { Phase }\end{array}$ & $\begin{array}{c}\text { Slow Expansion } \\
\left(a \sim(-t)^{n}, n \rightarrow 0_{-}\right)\end{array}$ & $\begin{array}{c}\text { Slow Contraction } \\
\left(a \sim(-t)^{n}, n \rightarrow 0_{+}\right)\end{array}$ & $\begin{array}{l}\text { Contraction } \\
\left(a \sim(-t)^{\frac{2}{3}}\right)\end{array}$ \\
\hline Ref. & & {$[9,10,17]$} & $4,5,6$ \\
\hline
\end{tabular}
Region I-IV in Table I. For $\zeta$ spectrum, stable Regions I, II, IV are dual to unstable Region III, and for $\Phi$ spectrum, stable Region I is self-dual and stable Region II is dual to unstable Region III and stable Region IV.

TABLE II: The dualities of nearly scale-invariant spectra: For $\Phi$ spectrum, the slowly expanding phase with $\epsilon \rightarrow-\infty$ (The relation between $\epsilon$ and $n$ can be seen from Eq. (7)) is dual to the phantom inflationary phase with $\epsilon=0_{-}$, in which the nearly scale-invariant blue spectrum is given, and the slowly contracting phase with $\epsilon \rightarrow \infty$ is dual to the usual inflationary phase with $\epsilon=0_{+}$, in which the nearly scale-invariant red spectrum is given. For $\zeta$ spectrum, the inflationary phase with $\epsilon \simeq 0$ is dual to the unstable contracting phase with $\epsilon \simeq \frac{3}{2}$.

expanding phase $(0 \leq \epsilon<1)$ to the contracting phase $\left(1<\epsilon \leq \frac{3}{2}\right)$, but the contracting phase with $\epsilon \geq 3$ is paired the contracting phase $(2<\epsilon \leq 3)$ and have no relevant expanding dual branch, while Boyle et.al.'s duality pairs the expanding phase $(0 \leq \epsilon<1)$ to the contracting phase $(1<\epsilon<\infty)$. Further, when relaxed $\epsilon$ to $\epsilon<0$, the dualities from various phases may be described more completely and can be regarded as the complementarity of Ref. [17]. In this case, Wands's duality pairs the expanding phase $(-\infty<\epsilon<0)$ to the contracting phase $\left(\frac{3}{2}<\epsilon<2\right)$, and Boyle et.al.'s duality pairs the slowly expanding phase $(-\infty<\epsilon \leq-1)$ to the expanding phase $(-1 \leq \epsilon<0)$.

The scale solution is a stable attractor for the expanding phase if and only if $w<1(\epsilon<3)$, and for the contracting phase if and only if $w>1(\epsilon>3)$ 9, 29]. Thus Wands's duality relates the stable expanding/contracting solutions to the unstable contracting solutions, while 
Boyle et.al.'s duality relates two stable branches only when $\epsilon<1 / 3$ or $\epsilon>3$, in terms of the spectrum index, which requires $n_{\Phi, \zeta}>0$. for $1 / 3<\epsilon<3$ the stable expanding solution is related to the unstable contracting branch, in this case $n_{\Phi, \zeta}<0$.

Due to these dualities, the primordial scalar perturbation spectra may be not used to determine in which phase the perturbations are generated. However, this degenerations may be remove by the tensor perturbation spectrum, whose spectrum index is 17]

$$
n_{t}=3-\left|\frac{\epsilon-3}{\epsilon-1}\right|
$$

The phase with $h$ rapidly increasing will produce a more blue tensor spectrum than its dual phase with $h$ slowly increasing or decreasing. The figure of the tensor perturbation spectrum with $w$ can be seen in Ref. [20]. For $\zeta$ spectrum, its scalar spectrum index is the same as that of tensor, but fortunately, in this case, for arbitrary stable phase, its stable dual branch does not exist. As a result if the spectrum is generated by a stable solution then the detection of the primordial gravitational wave background can give a direct record of the evolution of the scale factor, thus a direct selection for different ex- panding/contracting phases.

In summary, we discuss the dualities of the primordial perturbation spectra for full space of constant $w$ (thus $\epsilon)$. The results are consistent with Boyle et.al.'s for the case of $\epsilon>0$ [17]. The exist of these dualities means that various cosmological background solutions are dual each other in the sense of scalar perturbation spectrum, see Fig.2 for a sketch of dualities of various phases. Further, the nearly scale-invariant spectrum can be from various cosmological scenarios is not also coincidental, the relationship between them can be regarded as some special cases of general dualities of $\Phi$ or $\zeta$ spectrum, see Table II for a summary on the dualities of nearly scale-invariant spectra, which may provide a possibility that the perturbations of background solutions with $|\epsilon|>1$ can be studied by using slow-roll $(|\epsilon|<1)$ method [9, 34]. In this brief report, we only focus on the case of the single (nor$\mathrm{mal} /$ phantom) scalar field with exponential potential in which $w$ is constant and $c_{s}^{2}=1$. The studies of relaxing these limits may be more interesting.

Acknowledgments The author would like to thank Paul J. Steinhardt for helpful discussions and comments on the manuscript. This work is supported by K.C.Wang Postdoc Foundation.
[1] A.H. Guth, Phys. Rev. D23 (1981) 347; A.D. Linde, Phys. Lett. B108 (1982) 389; A.A. Albrecht and P.J. Steinhardt, Phys. Rev. Lett. 48 (1982) 1220.

[2] A.D. Linde, hep-th/0402051

[3] V. Mukhanov and G. Chibisov, JETP 33549 (1981); A.H. Guth and S.-Y. Pi, Phys. Rev. Lett. 49, 1110 (1982); S.W. Hawking, Phys. Lett. B115,295 (1982); A.A. starobinsky, Phys. Lett. B117 175 (1982); J.M. Bardeen, P.J. Steinhardt and M.S. Turner, Phys. Rev. D28 679 (1983).

[4] D. Wands, Phys. Rev. D60 023507 (1999).

[5] R. Brandenberger and F. Finelli, JHEP 0111, 056 (2001); F. Finelli and R. Brandenberger, hep-th/0112249

[6] A.A. Starobinsky, JETP Lett. 30682 (1979).

[7] M. Gasperini and G. Veneziano, Astropart. Phys. 1 (1993) 317, hep-th/9211021

[8] G. Veneziano, hep-th/0002094 J.E. Lidsey, D. Wands and E.J. Copeland, Phys. Rept. 337 (2000) 343, hep-th/9909061

[9] S. Gratton, J. Khoury, P.J. Steinhardt and N. Turok, astro-ph/0301395

[10] J. Khoury, P.J. Steinhardt and N. Turok, astro-ph/0302012

[11] J. Khoury, B.A. Ovrut, P.J. Steinhardt and N. Turok, Phys. Rev. D66 (2002) 046005.

[12] A.J. Tolley and N. Turok, Phys. Rev. D66 106005 (2002); A.J. Tolley, N. Turok and P.J. Steinhardt, hep-th/0306109

[13] J. Khoury, B.A. Ovrut, P.J. Steinhardt and N. Turok, Phys. Rev. D64 (2001) 123522; J. Khoury, B.A. Ovrut, N. Seiberg, P.J. Steinhardt and N. Turok, Phys. Rev. D65 (2002) 086007; P.J. Steinhardt and N. Turok, Sci- ence 296, (2002) 1436; Phys. Rev. D65 126003 (2002).

[14] J. Hwang, astro-ph/0109045 D.H. Lyth, Phys. Lett. B526,173 (2002); S. Tsujikawa, Phys. Lett. B526, 179 (2002); J. Martin, P. Peter, N. Pinto-Neto and D.J. Schwarz, Phys. Rev. D65, 123513 (2002).

[15] R. Kallosh, L. Kofman and A. Linde, Phys. Rev. D64 123523 (2001); R. Kallosh, L. Kofman, A. Linde and A. Tseytlin, Phys. Rev. D64, 123524 (2001).

[16] S. Tsujikawa, R. Brandenberger and F. Finelli, Phys. Rev. D66, 083513 (2002).

[17] L.A. Boyle, P.J. Steinhardt and N. Turok, hep-th/0403026

[18] Y.S. Piao and E Zhou, Phys. Rev. D68, 083515 (2003).

[19] Y.S. Piao and Y.Z. Zhang, Phys. Rev. D70, 063513 (2004), astro-ph/0401231

[20] Y.S. Piao and Y.Z. Zhang, Phys. Rev. D70, 043516 (2004), astro-ph/0403671

[21] L. Kofman, A.D. Linde and A.A. Starobinski, Phys. Rev. Lett. 733195 (1994); Phys. Rev. D56 3258 (1997).

[22] G.N. Felder, L. Kofman and A.D. Linde, Phys. Rev. D59 123523 (1999); Phys. Rev. D60 103505 (1999).

[23] L.F. Abbott and M.F. Wise, Nucl. Phys. B244, 541 (1984).

[24] J. Garriga and V.F. Mukhanov, Phys. Lett. B458 219 (1999).

[25] V.N. Lukash, JETP 79, 1601 (1980).

[26] V.F. Mukhanov, JETP lett. 41, 493 (1985); Sov. Phys. JETP. 68, 1297 (1988).

[27] H. Kodama and M. Sasaki, Prog. Theor. Phys. Suppl. 78 1 (1984).

[28] V.F. Mukhanov, H.A. Feldman and R.H. Brandenberger, Phys. Rept. 215, 203 (1992). 
[29] J.K. Erickson, D.H. Wesley, P.J. Steinhardt, N. Turok, hep-th/0312009

[30] C. Cartier, R. Durrer and E.J. Copeland, hep-th/0301198

[31] J. Martin and P. Peter, hep-th/0307077

[32] P. Peter and N. Pinto-Neto, hep-th/0203013 P. Peter,
N. Pinto-Neto and D.A. Gonzalez, hep-th/0306005

[33] L.E. Allen and D. Wands, astro-ph/0404441

[34] J. Khoury, P.J. Steinhardt and N. Turok, Phys. Rev. Lett. 92031302 (2004). 